\title{
Intravenous Thrombolysis in Patients Dependent on the Daily Help of Others Before Stroke
}

\author{
Henrik Gensicke, MD; Daniel Strbian, MD; Sanne M. Zinkstok, MD, PhD; \\ Jan F. Scheitz, MD; Olivier Bill, MD; Christian Hametner, MD; Solène Moulin, MD, MSc; \\ Andrea Zini, MD; Georg Kägi, MD; Alessandro Pezzini, MD; Visnja Padjen, MD, PhD; \\ Yannick Béjot, MD; Sydney Corbiere, MD; Thomas P. Zonneveld, MD; David J. Seiffge, MD; \\ Yvo B. Roos, MD, PhD; Christopher Traenka, MD; Jukka Putaala, MD; Nils Peters, MD; \\ Leo H. Bonati, MD; Sami Curtze, MD, PhD; Hebun Erdur, MD; Gerli Sibolt, MD; \\ Peter Koch, MD; Laura Vandelli, MD; Peter Ringleb, MD; Didier Leys, MD; \\ Charlotte Cordonnier, MD; Patrik Michel, MD; Christian H. Nolte, MD; Philippe A. Lyrer, MD; \\ Turgut Tatlisumak, MD; Paul J. Nederkoorn, MD, PhD; Stefan T. Engelter, MD; \\ for the Thrombolysis in Stroke Patients (TriSP) Collaborators
}

Background and Purpose-We compared outcome and complications in patients with stroke treated with intravenous thrombolysis (IVT) who could not live alone without help of another person before stroke (dependent patients) versus independent ones.

Methods-In a multicenter IVT-register-based cohort study, we compared previously dependent (prestroke modified Rankin Scale score, 3-5) versus independent (prestroke modified Rankin Scale score, 0-2) patients. Outcome measures were poor 3-month outcome (not reaching at least prestroke modified Rankin Scale [dependent patients]; modified Rankin Scale score of 3-6 [independent patients]), death, and symptomatic intracranial hemorrhage. Unadjusted and adjusted odds ratios (ORs) with 95\% confidence intervals (OR [95\% confidence interval]) were calculated.

Results-Among 7430 IVT-treated patients, 489 (6.6\%) were dependent and 6941 (93.4\%) were independent. Previous stroke, dementia, heart, and bone diseases were the most common causes of preexisting dependency. Dependent patients were more likely to die $\left(\mathrm{OR}_{\text {unadiusted }} 4.55\right.$ [3.74-5.53]; $\mathrm{OR}_{\text {adjusted }} 2.19$ [1.70-2.84]). Symptomatic intracranial hemorrhage occurred equally frequent (4.8\% versus $4.5 \%$ ). Poor outcome was more frequent in dependent (60.5\%) than in independent (39.6\%) patients, but the adjusted ORs were similar $\left(\mathrm{OR}_{\text {adjusted }}, 0.95\right.$ [0.75-1.21]). Among survivors, the proportion of patients with poor outcome did not differ (35.7\% versus 31.3\%). After adjustment for age and stroke severity, the odds of poor outcome were lower in dependent patients $\left(\mathrm{OR}_{\text {adjusted }}, 0.64[0.49-0.84]\right)$.

Conclusions-IVT-treated stroke patients who were dependent on the daily help of others before stroke carry a higher mortality risk than previously independent patients. The risk of symptomatic intracranial hemorrhage and the likelihood of poor outcome were not independently influenced by previous dependency. Among survivors, poor outcome was avoided at least as effectively in previously dependent patients. Thus, withholding IVT in previously dependent patients might not be justified. (Stroke. 2016;47:450-456. DOI: 10.1161/STROKEAHA.115.011674.)

Key Words: infusions, intravenous $\boldsymbol{\square}$ intracranial hemorrhages $\mathbf{m}$ outcome assessment (health care)

- stroke — survivors

Received September 29, 2015; final revision received December 2, 2015; accepted December 7, 2015.

From the Stroke Center and Department of Neurology, University Hospital Basel, Basel, Switzerland (H.G., S.C., D.J.S., C.T., N.P., L.H.B., P.A.L., S.T.E.); Department of Neurology, Helsinki University Central Hospital, Helsinki, Finland (D.S., J.P., S.C., G.S., T.T.); Department of Neurology, Academic Medical Center, Amsterdam, The Netherlands (S.M.Z., T.P.Z., Y.B.R., P.J.N.); Department of Neurology and Center for Stroke Research, Charité-Universitätsmedizin Berlin, Berlin, Germany (J.F.S., H.E., P.K., C.H.N.); Department of Neurology, Centre Hospitalier Universitaire Vaudois and University of Lausanne, Lausanne, Switzerland (O.B., P.M.); Department of Neurology, University Hospital Heidelberg, Heidelberg, Germany (C.H., P.R.); University Lille, Inserm, CHU Lille, U1171-Degenerative and Vascular Cognitive Disorders, Lille, France (S.M., D.L., C.C.); Stroke Unit, Department of Neuroscience, Nuovo Ospedale Civile S. Agostino-Estense, AUSL Modena, Modena, Italy (A.Z., L.V.); Department of Neurology, Kantonsspital St. Gallen, St Gallen, Switzerland (G.K.); Department of Clinical and Experimental Sciences, Neurology Clinic, University of Brescia, Brescia, Italy (A.P.); Department of Neurology, Clinical Centre of Serbia, Beograd, Serbia (V.P.); Department of Neurology, University Hospital, and Dijon Stroke Registry, University of Burgundy, Dijon, France (Y.B.); Department of Neurology, Sahlgrenska University Hospital, Gothenburg, Sweden (T.T.); and Department for Medicine of Aging and Rehabilitation, University Center, Felix Platter Hospital, Basel, Switzerland (S.T.E.).

The online-only Data Supplement is available with this article at http://stroke.ahajournals.org/lookup/suppl/doi:10.1161/STROKEAHA.115. 011674/-/DC1.

Correspondence to Henrik Gensicke, MD, Department of Neurology, University Hospital Basel, Petersgraben 4, CH-4031 Basel, Switzerland. E-mail henrik.gensicke@usb.ch

(C) 2016 American Heart Association, Inc.

Stroke is available at http://stroke.ahajournals.org

DOI: 10.1161/STROKEAHA.115.011674 
$\mathrm{I}_{\mathrm{p}}^{\mathrm{n}}$ ntravenous thrombolysis (IVT) is efficacious and safe in patients with acute ischemic stroke. ${ }^{1}$ However, it is unclear whether this is also true for patients who could not live alone without daily help of another person prior to stroke (ie, modified Rankin Scale score of $\geq 3){ }^{2}$ Such patients were not eligible for the large controlled randomized IVT trials including third international stroke trial (IST-3). ${ }^{3}$ In clinical practice, some patients with preexisting dependency receive IVT, ${ }^{4}$ whereas others do not. ${ }^{5}$ The present study aimed at investigating the impact of preexisting dependency on outcomes and (bleeding) complications in a large population of IVT-treated stroke patients.

\section{Methods}

As a joint initiative of 12 European stroke centers, the Thrombolysis in Ischemic Stroke Patients (TriSP) investigators performed a large collaborative clinical cohort study.

All participating centers treated patients with acute ischemic stroke with IVT according to current guidelines (http://www.esostroke.org/eso-stroke/education/guidelines.html).

Data from individual patients were collected with a standardized form with predefined variables as it was done in previous studies. ${ }^{6-9}$ Local study investigators filled in the forms systematically using prospectively ascertained in-hospital intravenous ischemic stroke thrombolysis registries. Completed forms from all centers were compiled in the coordinating center Basel, where the analysis of the pooled data was performed, as done previously. ${ }^{6,7}$ The study was approved by the ethics committee in Basel, Switzerland. The requirement for additional local ethical approval differed between participating centers and was obtained if required.

The following prospectively ascertained variables were used: age, sex, modified Rankin Scale before stroke (prestroke mRS), initial stroke severity as assessed by the National Institutes of Health Stroke Scale score, ${ }^{10}$ blood pressure before IVT, onset-to-treatment time, renal function as quantified by the estimated glomerular filtration rate (Chronic Kidney Disease Epidemiology Collaboration ${ }^{7}$ ) and glucose levels in blood serum, vascular risk factors according to predefined criteria, ${ }^{11}$ and previous treatment with antithrombotic agents (antiplatelet agents or anticoagulants). Prestroke mRS score had been estimated by the stroke physicians who also indicated and applied IVT. The scores were based on information provided by patients and next-to-kin during the information about benefits and risks of IVT, and all written reports available before IVT. Functional 3-month outcome was assessed with the mRS by using a structured interview at outpatient visits or telephone calls with patients or relatives. There was monitoring for intracranial hemorrhage (ICH) by follow-up computed tomography or magnetic resonance imaging as done in previous research. ${ }^{6,7}$

Each center reported on the period for which they had prospectively collected data on consecutive patients up to November 30, 2014 (Table I in the online-only Data Supplement). All patients with missing prestroke mRS and missing 3-month outcome data were excluded. Preexisting dependency was defined by a prestroke mRS score of 3 to 5 , while preexisting independency meant a prestroke mRS score of 0,1 , or 2 .

Outcome and complication measures were as follows: (1) poor functional 3-month outcome, defined as 3-month mRS score of 3 to 6 in previously independent patients and a 3-month mRS higher than the prestroke mRS in previously dependent patients as applied in previous research ${ }^{12}$ and shown in Figure 1, (2) death, and (3) symptomatic ICH ( $\mathrm{sICH})$ according to criteria of the European Cooperative Acute Stroke Study (ECASS)-II trial. ${ }^{13}$

\section{Main Analysis}

We investigated the impact of preexisting dependency on outcomes by comparing patients who were dependent on the help of others before stroke (prestroke mRS score, 3-5) with previously independent patients (prestroke mRS score, 0-2; Figure 1).

\section{Subgroup Analyses}

First, we investigated the impact of different degrees of prestroke dependency on outcomes. Patients who were dependent on the daily help of others before stroke were subdivided in those with complete dependency (ie, prestroke mRS score, 4-5) and in those patients with partial dependency (ie, prestroke mRS score, 3; Figure 1). Second, among survivors at 3 months, we studied the impact of prestroke dependency on functional outcome. Third, in a subset of patients, the causes of preexisting dependency were available from the following centers: Amsterdam, Basel, Berlin, Brescia, and Modena. The diagnosis of comorbidities causing dependency was provided by local investigators and categorized by consensus by three adjudicators (H.G., S.C., S.T.E.)

\section{Statistical Analyses}

Statistical analysis was performed using SPSS (version 19.0 for Windows; SPSS Inc, Chicago, IL).

For the main analysis, patient's prestroke mRS was divided into 2 groups: prestroke mRS score of 0 to 2 and prestroke mRS score of 3 to 5. Prestroke mRS score of 0 to 2 served as the reference group. For subgroup analyses, previously dependent patients were subdivided into prestroke mRS score of 3 (partial dependency) and mRS score of 4 to 5 (complete dependency). Data were summarized as median ( \pm interquartile range). We used Fisher exact test, $\chi^{2}$ test for categorical variables and the Mann-Whitney $U$ test for continuous variables, where appropriate. The association between prestroke $\mathrm{mRS}$ and outcome was estimated by calculating odds ratios (OR) with $95 \%$ confidence intervals (95\% CIs), using binary logistic regression models. In the multivariable analysis, the models were adjusted for all variables with $P<0.1$ in the univariable analyses.

\section{Results}

\section{Baseline Characteristics}

Data were eligible for analysis in $7430(89.2 \%)$ of the 8327 IVT-treated patients. Six-hundred fifty-nine $(7.9 \%)$ patients were excluded from analyses because of missing prestroke mRS data and 238 (2.9\%) because of missing 3-month outcome data. Among study patients, 6941 (93.4\%) were previously independent and 489 (6.6\%) dependent before stroke. The latter included 347 patients with prestroke mRS score of 3,132 with prestroke mRS score of 4 , and 10 patients with prestroke mRS score of 5. Previously dependent patients were older, more often women, had more severe strokes, more often vascular risk factors (except smoking and hypercholesterolemia), and were more often on antithrombotic medication than previously independent patients (Table 1). The distribution of 3-month mRS depending on prestroke mRS is presented in Figure 2A.

In 5 centers, data on causes of preexisting dependency were available. In 193 patients, preexisting dependency was caused by a single disease in $133(68.9 \%)$ patients and multifactorial in $60(31.1 \%)$ patients. Causes of preexisting dependency included dementia $(\mathrm{n}=88 ; 45.6 \%)$, previous strokes $(\mathrm{n}=32 ; 16.6 \%)$, heart diseases $(\mathrm{n}=32 ; 16.6 \%)$, and degenerative or traumatic bone diseases $(\mathrm{n}=38 ; 19.7 \%)$. Details are shown in Table II in the online-only Data Supplement.

\section{Logistic Regression Analyses}

In univariable analyses, poor outcome (60.5\% versus $39.6 \%)$ and death (38.7\% versus $12.2 \%$ ) occurred significantly more often in patients with previous dependency than in independent patients. sICH (4.8\% versus $4.5 \%)$ occurred equally 
A

Pre-stroke functional status (pre-stroke mRS)
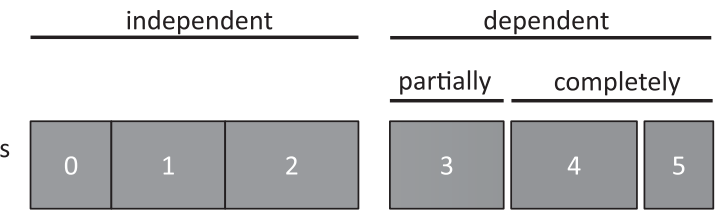

B

Pre-stroke functional status

independent

(pre-stroke mRS 0-2)

partially dependent

Poor functional outcome at 3 months (pre-stroke mRS 3)

completely dependent (pre-stroke mRS 4)

completely dependent (pre-stroke mRS 5)
Figure 1. Definition of (A) prestroke functional status and (B) poor functional outcome at 3 mo. mRS indicates modified Rankin Scale. often (Table 2; Figure 2B). After adjustment for potentially confounding variables, previously dependent patients were at increased risk of death $\left(\mathrm{OR}_{\text {adjusted }}, 2.19 ; 95 \% \mathrm{CI}, 1.70-2.84\right.$; $P<0.001)$ but not for poor outcome $\left(\mathrm{OR}_{\text {adjusted }}, 0.95 ; 95 \% \mathrm{CI}\right.$, 0.75-1.21). The occurrence of sICH did not differ significantly between both groups (Tables 3 and 4).

\section{Different Degrees of Dependency}

Patients with complete dependency before stroke (prestroke mRS score, 4 or 5) had a higher median National Institutes
$\mathrm{mRS}$ at 3 months

\begin{tabular}{r|r|l|}
\hline 3 & 5 & 6 \\
\hline 4 & 5 & 6 \\
\hline 5 & 6 \\
& 6 \\
\hline
\end{tabular}

of Health Stroke Scale on admission than patients with partial dependency (prestroke mRS score, 3; Table 1). They died significantly more often within 3 months after IVT $(48.6 \%$ versus $34.6 \%$ ), whereas all other outcome events did not differ significantly between both groups (Table 2; Figure 2B). In regression analyses, the increased likelihood of death for those with complete dependency was similar with and without adjustment $\left(\mathrm{OR}_{\text {unadjusted }}, 1.79 ; 95 \% \mathrm{CI}, 1.20-2.66 ; P=0.004\right.$ and $\left.\mathrm{OR}_{\text {adjusted }}, 1.72 ; 95 \% \mathrm{CI}, 1.11-2.65 ; P=0.014\right)$. The OR for poor outcome and $\mathrm{sICH}$ did not differ significantly between

Table 1. Clinical Characteristics of Intravenous Thrombolysis-Treated Stroke Patients Divided Into Groups Depending on Their Prestroke Functional Status

\begin{tabular}{|c|c|c|c|c|c|c|}
\hline & $\begin{array}{l}\text { Prestroke Independent } \\
\qquad(\mathrm{n}=6941)\end{array}$ & $\begin{array}{l}\text { Prestroke Dependent } \\
\qquad(\mathrm{n}=489)\end{array}$ & $\begin{array}{c}\text { Independent vs } \\
\text { Dependent ( } P \text { Value) }\end{array}$ & $\begin{array}{l}\text { Prestroke Partially } \\
\text { Dependent }(n=347)\end{array}$ & $\begin{array}{c}\text { Prestroke Completely } \\
\text { Dependent }(n=142)\end{array}$ & $\begin{array}{c}\text { Partially vs } \\
\text { Completely ( } P \text { Value) }\end{array}$ \\
\hline Age, y, median (IQR) & 71 (60-79) & $84(77-88)$ & $<0.001$ & $84(77-88)$ & 84 (76-89) & 0.786 \\
\hline Men, n (\%) & $3983(57.4)$ & $166(33.9)$ & $<0.001$ & $121(34.9)$ & $45(31.7)$ & 0.529 \\
\hline $\begin{array}{l}\text { Stroke severity, NIHSS, } \\
\text { median (IQR) }\end{array}$ & $10(6-16)$ & $14(8-19)$ & $<0.001$ & $13(8-19)$ & $17(10-20)$ & 0.009 \\
\hline $\begin{array}{l}\text { Systolic blood pressure, } \\
\mathrm{mm} \mathrm{Hg} \text {, median (IQR) }\end{array}$ & $155(140-170)$ & $154(140-173)$ & 0.558 & $154(140-171)$ & $159(139-175)$ & 0.808 \\
\hline $\begin{array}{l}\text { Onset-to-treatment, } \\
\text { min, median (IQR) }\end{array}$ & $145(105-180)$ & $140(107-192)$ & 0.278 & $140(107-200)$ & $145(107-190)$ & 0.810 \\
\hline $\begin{array}{l}\text { GFR, mL/min per } 1.73 \\
\mathrm{~m}^{2}, \text { median (IQR) }\end{array}$ & 78 (60-92) & $58(43-78)$ & $<0.001$ & $59(45-79)$ & $57(40-75)$ & 0.177 \\
\hline $\begin{array}{l}\text { Glucose on admission, } \\
\mathrm{mmol} / \mathrm{L} \text {, median (IQR) }\end{array}$ & $6.6(5.7-7.9)$ & $6.7(5.8-8.3)$ & 0.031 & $6.8(5.8-8.3)$ & $6.6(5.8-8.0)$ & 0.181 \\
\hline Atrial fibrillation, $\mathrm{n}(\%)$ & $1733(25.0)$ & $225(46.1)$ & $<0.001$ & $160(46.2)$ & $65(45.8)$ & 0.925 \\
\hline Hypertension, n (\%) & $4563(65.9)$ & $386(79.1)$ & $<0.001$ & $275(79.5)$ & $111(78.2)$ & 0.806 \\
\hline Smoking, n (\%) & $1362(22.8)$ & $37(8.1)$ & $<0.001$ & $30(9.3)$ & $7(5.2)$ & 0.187 \\
\hline $\begin{array}{l}\text { Hypercholesterolemia, } \\
\text { n (\%) }\end{array}$ & $2942(42.5)$ & $184(38.3)$ & 0.086 & $139(40.9)$ & $45(32.1)$ & 0.079 \\
\hline Diabetes mellitus, n (\%) & $1220(17.6)$ & $126(25.8)$ & $<0.001$ & 95 (27.5) & $31(21.8)$ & 0.212 \\
\hline $\begin{array}{l}\text { Coronary artery } \\
\text { disease, } \mathrm{n}(\%)\end{array}$ & $1192(17.3)$ & $115(23.6)$ & 0.001 & 79 (22.9) & $36(25.4)$ & 0.559 \\
\hline Previous stroke, n (\%) & $968(14.0)$ & $157(32.3)$ & $<0.001$ & $103(29.9)$ & $54(38.0)$ & 0.089 \\
\hline $\begin{array}{l}\text { Previous } \\
\text { antithrombotics, n (\%) }\end{array}$ & $2480(36.1)$ & $310(64.7)$ & $<0.001$ & $222(55.1)$ & $88(63.8)$ & 0.833 \\
\hline
\end{tabular}

GFR indicates glomerular filtration rate; mRS, modified Rankin Scale; NIHSS, National Institutes of Health Stroke Scale; prestroke completely dependent, prestroke $\mathrm{mRS}$ score of 4 to 5; prestroke dependent, prestroke mRS score of 3 to 5 ; prestroke independent, prestroke mRS score of 0 to 2; and prestroke partially dependent, prestroke mRS score of 3 . 
A

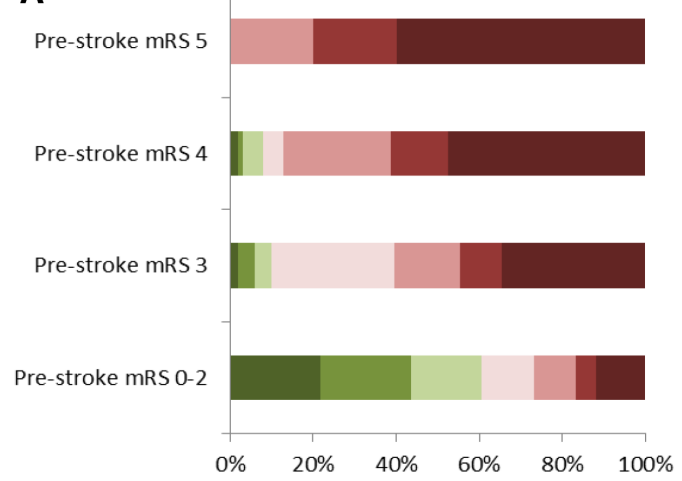

B

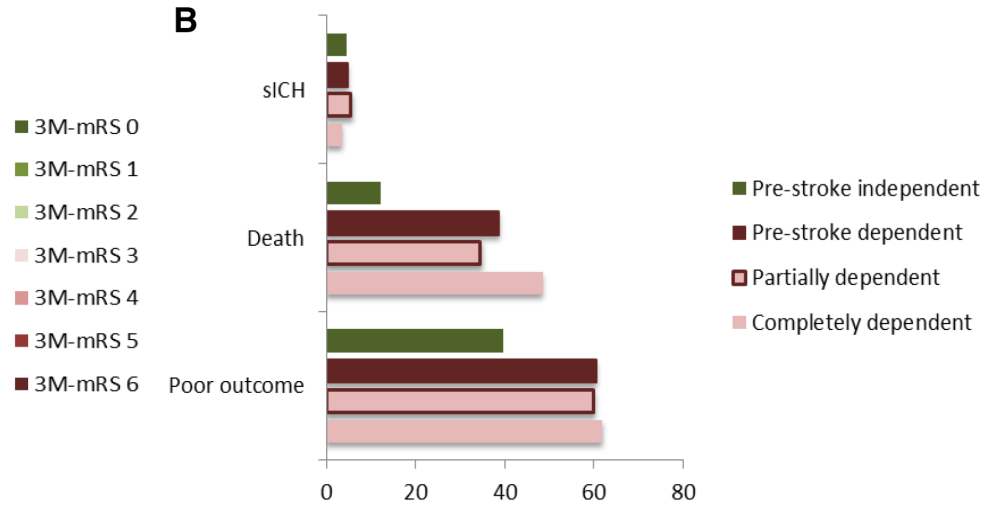

Figure 2. Distribution of 3-mo modified Rankin Scale (mRS) depending on prestroke mRS $(\mathbf{A})$ and frequency of symptomatic intracranial hemorrhage, death or poor outcome at $3 \mathrm{mo}(\mathbf{B})$ - stratified to the dependency level before stroke. Data are presented in percent of all intravenous thrombolysis-treated stroke patients of each group. 3M-mRS indicates mRS at 3 mo; poor outcome, not reaching at least prestroke mRS in previously dependent patients (mRS score of 3 to 6 in previously independent patients); prestroke independent, prestroke mRS score of 0 to 2; prestroke dependent, prestroke mRS score of 3 to 5; prestroke partially dependent, prestroke mRS score of 3; prestroke completely dependent, prestroke mRS score of 4 to 5; and sICH, symptomatic intracranial hemorrhage (European Cooperative Acute Stroke Study [ECASS] II definition).

patients with complete versus partial dependency before stroke (Tables 3 and 4).

\section{Outcome of Survivors}

Among survivors, the proportion of patients with poor functional outcome did not differ significantly between previously dependent and independent patients (35.7\% versus 31.3\%; $\left.\mathrm{OR}_{\text {unadiusted }}, 1.22 ; 95 \% \mathrm{CI}, 0.96-1.55 ; P=0.112\right)$. However, after adjustment for age and National Institutes of Health Stroke Scale, the likelihood for poor outcome was lower in previously dependent patients $\left(\mathrm{OR}_{\text {adjusted }}, 0.64 ; 95 \% \mathrm{CI}, 0.49-0.84\right.$; $P=0.001$ ).

\section{Discussion}

This exploratory multicenter study addressed the impact of preexisting dependency on outcome and complications in IVTtreated stroke patients. We observed the following key findings: (1) previously dependent patients had a higher 3-month mortality rate than previously independent patients. (2) The frequency of symptomatic ICH was similar in both groups. Among survivors, previously dependent patients had higher odds to avoid poor 3-month outcome than independent ones if age and stroke severity were taken into account. (4) Causes of preexisting dependency were multifactorial in one third of patients and mostly caused by dementia, previous stroke, heart disease, and degenerative or traumatic bone diseases.
In our cohort, $\approx 40 \%$ of the IVT-treated patients with preexisting disability had died within 3 months. This rate is comparable with previous observations in smaller studies, in which 7 of 14 patients, and $49 \%$ among 171 patients with previous dependency (applying the same definition as in our cohort) died within 3 months. ${ }^{4,12}$ As a refinement, our data allowed an estimate of the mortality risk. After adjustment for confounding variables, being previously dependent meant that the likelihood was twice as high to die within the next 3 months after IVT when compared with previously independent patients treated with IVT.

In a non-IVT-treated stroke population, preexisting disability was independently associated with death with an OR of 2.1 (95\% CI, 1.4-3.2), ${ }^{14}$ virtually identical to that in our IVTtreated patients $\left(\mathrm{OR}_{\text {adjusted }}, 2.19 ; 95 \% \mathrm{CI}, 1.70-2.84\right)$.

The observed higher mortality is not explained by an excess of bleeds, as sICH occurred as often in dependent as in independent patients. Possible explanations for the higher 3-month mortality among previously dependent patients may include (1) a higher susceptibility for complications other than sICH, (2) worsening of preexisting comorbid diseases triggered by the stroke, (3) a higher disease burden caused by preexisting comorbidities in such patients, and (4) withdrawal of care according to patient's preferences.

Furthermore, and as a novelty, our data showed that the mortality risk increased with increasing degree of preexisting dependency. For patients with preexisting complete dependency, the odds for being dead at 3 months were $\approx 10 \%$

Table 2. Outcome Events of Intravenous Thrombolysis-Treated Stroke Patients Divided Into Prestroke Modified Rankin Scale Groups

\begin{tabular}{lcccccc}
\hline & $\begin{array}{c}\text { Prestroke Independent } \\
(\mathrm{n}=6941)\end{array}$ & $\begin{array}{c}\text { Prestroke Dependent } \\
(\mathrm{n}=489)\end{array}$ & $\begin{array}{c}\text { Independent vs } \\
\text { Dependent }(P \text { Value })\end{array}$ & $\begin{array}{c}\text { Prestroke Partially } \\
\text { Dependent }(\mathrm{n}=347)\end{array}$ & $\begin{array}{c}\text { Prestroke Completely } \\
\text { Dependent }(\mathrm{n}=142)\end{array}$ & $\begin{array}{c}\text { Partially vs Completely } \\
(P \text { Value })\end{array}$ \\
\hline Poor outcome, $\mathrm{n}(\%)$ & $2751(39.6)$ & $296(60.5)$ & $<0.001$ & $208(59.9)$ & $88(62.0)$ & 0.677 \\
Death, $\mathrm{n}(\%)$ & $845(12.2)$ & $189(38.7)$ & $<0.001$ & $120(34.6)$ & $69(48.6)$ & 0.004 \\
sICH, $\mathrm{n}(\%)$ & $308(4.5)$ & $23(4.8)$ & 0.591 & $19(5.5)$ & $5(3.6)$ & 0.380 \\
\hline
\end{tabular}

mRS indicates modified Rankin Scale; poor outcome, not reaching at least prestroke mRS in previously dependent patients (mRS score of 3 to 6 in previously independent patients); prestroke completely dependent, prestroke mRS score of 4 to 5; prestroke dependent, prestroke mRS score of 3 of 5 ; prestroke independent, prestroke mRS score of 0 to 2; prestroke partially dependent, prestroke mRS score of 3; and sICH, symptomatic intracranial hemorrhage (European Cooperative Acute Stroke Study [ECASS] II definition). 
Table 3. Univariable Analysis of Clinical Characteristics (Odds Ratio With 95\% Confidence Interval) in Intravenous Thrombolysis-Treated Patients

\begin{tabular}{lccc}
\hline & \multicolumn{3}{c}{ Outcome Measures } \\
\cline { 2 - 4 } Putative Predicting Variables & Poor Outcome & Death & sICH \\
\hline Age (each y) & $1.04(1.04-1.05) ; P<0.001$ & $1.06(1.05-1.07) ; P<0.001$ & $1.03(1.02-1.03) ; P<0.001$ \\
Sex (women vs men) & $1.36(1.24-1.50) ; P<0.001$ & $1.29(1.13-1.47) ; P<0.001$ & $0.99(0.79-1.23) ; P=0.91$ \\
NIHSS (each point) & $1.16(1.15-1.17) ; P<0.001$ & $1.15(1.14-1.16) ; P<0.001$ & $1.08(1.06-1.09) ; P<0.001$ \\
Glucose (each mg/dL) & $1.08(1.06-1.10) ; P<0.001$ & $1.09(1.07-1.11) ; P<0.001$ & $1.04(1.02-1.07) ; P=0.002$ \\
GFR (each mL/min per 1.73 m²) & $1.02(1.02-1.02) ; P<0.001$ & $1.03(1.03-1.03) ; P<0.001$ & $1.01(1.01-1.02) ; P<0.001$ \\
Systolic blood pressure (each mm Hg) & $0.99(0.99-1.01) ; P=0.108$ & $0.99(0.99-1.01) ; P=0.075$ & $1.01(1.00-1.01) ; P=0.008$ \\
Onset-to-treatment (each min) & $1.01(1.00-1.02) ; P<0.001$ & $1.01(1.00-1.01) ; P<0.001$ & $1.01(1.00-1.01) ; P=0.031$ \\
Atrial fibrillation & $1.85(1.67-2.05) ; P<0.001$ & $1.99(1.73-2.28) ; P<0.001$ & $1.42(1.12-1.79) ; P=0.003$ \\
Diabetes mellitus & $1.41(1.26-1.59) ; P<0.001$ & $1.40(1.20-1.64) ; P<0.001$ & $1.33(1.02-1.73) ; P=0.037$ \\
Hypertension & $1.43(1.30-1.58) ; P<0.001$ & $1.56(1.34-1.81) ; P<0.001$ & $1.25(0.98-1.59) ; P=0.077$ \\
Hypercholesterolemia & $0.87(0.79-0.96) ; P=0.003$ & $0.85(0.74-0.97) ; P=0.017$ & $1.05(0.84-1.31) ; P=0.648$ \\
Current smoking & $0.64(0.56-0.72) ; P<0.001$ & $0.45(0.38-0.59) ; P<0.001$ & $0.70(0.51-0.96) ; P=0.028$ \\
Coronary artery disease & $1.36(1.20-1.53) ; P<0.001$ & $1.87(1.6-2.17) ; P<0.001$ & $1.35(1.03-1.75) ; P=0.029$ \\
Previous ischemic stroke & $1.24(1.09-1.40) ; P=0.001$ & $1.36(1.15-1.62) ; P<0.001$ & $1.12(0.88-1.58) ; P=0.280$ \\
Previous antithrombotic treatment & $1.38(1.26-1.52) ; P<0.001$ & $1.87(1.64-2.14) ; P<0.001$ & $1.36(1.01-1.70) ; P=0.007$ \\
Dependent vs independent & $2.34(1.94-2.82) ; P<0.001$ & $4.55(3.74-5.53) ; P<0.001$ & $0.89(0.58-1.36) ; P=0.591$ \\
Partially dependent vs independent & $2.28(1.83-2.84) ; P<0.001$ & $3.81(3.02-4.81) ; P<0.001$ & $1.26(0.78-2.03) ; P=0.35$ \\
Completely dependent vs independent & $2.48(1.76-3.50) ; P<0.001$ & $6.82(4.87-9.55) ; P<0.001$ & $0.80(0.33-1.97) ; P=0.627$ \\
Completely dependent vs partially & $1.09(0.73-1.63) ; P=0.677$ & $1.79(1.20-2.66) ; P=0.004$ & $0.64(0.23-1.74) ; P=0.38$ \\
dependent & & &
\end{tabular}

GFR indicates glomerular filtration rate; mRS, modified Rankin Scale; NIHSS, National Institutes of Health Stroke Scale; poor outcome, not reaching at least prestroke mRS in previously dependent patients (mRS score of 3 to 6 in previously independent patients); prestroke completely dependent, prestroke mRS score of 4 to 5 ; prestroke dependent, prestroke mRS score of 3 to 5 ; prestroke independent, prestroke mRS score of 0 to 2; prestroke partially dependent, prestroke mRS score of 3; and sICH, symptomatic intracranial hemorrhage (European Cooperative Acute Stroke Study [ECASS] II definition).

to $250 \%$ higher than in patients with partial dependency $\left(\mathrm{OR}_{\text {adjusted }}, 1.72 ; 1.11-2.65\right)$. Interestingly, the odds for poor functional outcome did not differ significantly between these 2 groups.

Patients with previous dependency more often had poor functional outcome than previously independent patients (60.5\% versus $39.6 \%)$. However, after adjustment for potential confounders preexisting dependency did not predict poor outcome independently ( $\left.\mathrm{OR}_{\text {adjusted }}, 0.95 ; 95 \% \mathrm{CI}, 0.75-1.21\right)$. This observation is unpreceded and differed from the result of a subgroup analysis in another study, suggesting a higher risk of poor outcome in patients with preexisting dependency $\left.\left(\mathrm{OR}_{\text {adjusted }}, 1.70 ; 0.99-2.94\right)\right)^{12}$

Unexpectedly, among survivors, previously dependent patients had better odds to avoid poor outcome than independent patients in our cohort if age and stroke severity were taken into account. We can only speculate on possible reasons: (1) our definition of poor outcome (which we adopted from a previous study ${ }^{12}$ ) differed between both groups and may have favored the previously dependent patients. In detail, among survivors, dependent patients with a prestroke mRS score of 3 can only move to an $\mathrm{mRS}$ score of 4 or 5 , patients with a

Table 4. Multivariable Analysis of Primary Outcomes (Odds Adjusted for All Variables With $P<0.1$ in the Univariable Analysis) in Intravenous Thrombolysis-Treated Patients

\begin{tabular}{lccc}
\hline & \multicolumn{3}{c}{ Outcome Measures } \\
\cline { 2 - 4 } Putative Predicting Variables & Poor Outcome & Death & sICH \\
\hline Dependent vs independent & $0.95(0.75-1.21) ; P=0.679$ & $2.19(1.70-2.84) ; P<0.001$ & $0.66(0.42-11.04) ; P=0.073^{*}$ \\
Partially dependent vs independent & $0.97(0.73-1.28) ; P=0.817$ & $1.75(1.30-2.37) ; P<0.001$ & $0.76(0.46-1.26) ; P=0.287^{*}$ \\
Completely dependent vs independent & $0.98(0.66-1.44) ; P=0.902^{*}$ & $3.11(2.09-4.4) ; P<0.001^{*}$ & $\ldots$ \\
Completely dependent vs partially dependent & $0.96(0.62-1.49) ; P=0.874^{*}$ & $1.72(1.11-2.65) ; P=0.014^{*}$ & $\ldots$ \\
\hline
\end{tabular}

Values represent odds ratio (95\% confidence interval); $P$ value. mRS indicates modified Rankin Scale; poor outcome, not reaching at least prestroke $m R S$ in previously dependent patients (mRS score of 3 to 6 in previously independent patients); prestroke completely dependent, prestroke mRS score of 4 to 5 ; prestroke dependent, prestroke mRS score of 3 of 5 ; prestroke independent, prestroke mRS score of 0 to 2; prestroke partially dependent, prestroke mRS score of 3; and sICH, symptomatic intracranial hemorrhage (European Cooperative Acute Stroke Study [ECASS] II definition).

*Adjusted for age, National Institutes of Health Stroke Scale only. 
prestroke mRS score of 4 only to mRS score of 5, and among survivors, patients with a prestroke mRS score of 5 cannot have a poor outcome. In contrast, previously independent have a greater capacity to have poor outcome (mRS score, 3-5). (2) The functional worsening caused by the current stroke (measured with the mRS) might be less severe in patients with an existing impairment of capability in activities of daily living as in patients without previous deficits. Thus, we urge to a cautious interpretation of this observation. (3) Theoretically, one might hypothesize that some causes of preexisting dependency (eg, depression, Parkinson disease, and bone fractures) might have improved during the 3-month follow-up with rehabilitative and medical therapy.

In previous research, the causes for preexisting disability or dependency were not addressed. ${ }^{4,12}$ In our cohort, causes were multiple in about one third of patients. Dementia, sequela from previous stroke, heart diseases, and degenerative or traumatic bone diseases were the most common causes for preexisting dependency. For dementia, 2 recent observational studies showed no significant differences in (in-hospital) mortality and occurrence of ICH between IVT-treated stroke patients with and without dementia. ${ }^{15,16}$ In turn, dementia requiring custodial care has been a reported exclusion criteria for IVT in some centers. ${ }^{17}$ For previous stroke, a large casecontrol study showed that such patients seem to benefit from IVT when compared with those who were not IVT treated. ${ }^{18}$ Thus, taking into account the aforementioned observations and our findings, it might be questionable to withhold IVT from acute stroke patients with dementia or sequela of previous strokes even if these comorbidities have had caused dependency before the current stroke.

Strengths of this study include (1) the relatively large sample size, which (a) reduces the risk of chance findings and (b) allows adjustment for (potentially) confounding variables; (2) a low number of missing data on 3-month outcome (3.1\%), which reduces the risk of bias, and (3) the systematic and standardized assessment of data, which were collected prospectively uninfluenced by the current research question.

Nevertheless, we are aware of several limitations: (1) data came from registries that were not monitored and nonrandomized. Furthermore, the low frequency of patients with preexisting dependency (ie, 6.6\%) in our IVT-cohort suggests a selection bias. We do not know how many dependent patients were not treated with IVT. More importantly, we do not know how dependent patients treated with IVT differed from those who did not receive IVT. However, it may be assumed that preferentially those dependent patients got IVT, who had a favorable prognosis. Thus, the outcomes detected in our IVT-treated sample of dependent patients should not be considered representative of all dependent patients who might be otherwise eligible for IVT. In addition, in $\approx 8 \%$ of the patients, information about prestroke mRS was missing. Thus, because of these limitations, we urge to a cautious interpretation of our results. (2) We compared previously dependent with previously independent patients who both had received IVT. To answer the question whether IVT is effective and safe in patients with preexisting dependency, a randomized controlled trial comparing IVT versus non-IVT in such patients would be required. At least our results indicate that such a trial would be ethically justified. (3) Providing outcome data separately for the subset of survivors involves the risk that outcomes might be perceived inappropriately good. In clinical practice, patients (and relatives) often do fear to be alive but dependent more than to die after stroke. (4) The assessment of the prestroke mRS is challenging, and its reliability is limited. ${ }^{19}$ Furthermore, the raters of the mRS at 3 months were not blinded to the prestroke mRS, which may have had influenced their rating. (5) The meaning of the causes of preexisting dependency could not be addressed sufficiently because such data were only available for a subgroup of patients.

\section{Conclusions}

The present study revealed that patients with IVT-treated stroke who were dependent on the daily help of others before stroke carry a higher mortality risk than previously independent patients. However, the risk of sICH was not different between these 2 groups, suggesting that IVT is apparently safe in this group. Among survivors, the adjusted odds to avoid poor outcome were even higher in previously dependent patients. Thus, withholding IVT in previously dependent patients might not be justified.

\section{Acknowledgments}

Dr Gensicke designed/conceptualized the study, analyzed/interpreted the data, drafted the article, and collected data. Drs Strbian, Zinkstok, Scheitz, Bill, Hametner, Moulin, Zini, Kägi, Pezzini, Padjen, Béjot, Corbiere, Zonneveld, Seiffge, Roos, Traenka, Putaala, Peters, Bonati, Curtze, Erdur, Sibolt, Koch, Vandelli, Ringleb, Leys, Cordonnier, Michel, Nolte, Lyrer, Tatlisumak revised the article, collected, and interpreted the data. Dr Nederkoorn designed/conceptualized and initiated the study, revised the article, collected, and interpreted the data. Dr Engelter designed/conceptualized and initiated the study, supervised the study, analyzed/interpreted the data, revised the article, and collected data.

\section{Sources of Funding}

This research was partly supported by the Stroke-[Hirnschlag]-Fund Basel and by grants from the Swiss National Foundation (33CM30124119 and 33CM30-140340/1) and the University of Basel.

\section{Disclosures}

Dr Seiffge received research grant from Swiss Heart Foundation; advisory board: Bayer. Dr Kägi received modest honoraria for travel and advisory board from Bayer and Nestle. Dr Béjot served as member of a scientific board for Boehringer-Ingelheim France, and received speaker honoraria from Astra-Zeneca. Dr Putaala received modest honoraria for lectures and advisory board from BoehringerIngelheim. Dr Peters has received travel honoraria from BoehringerIngelheim as well honoraria for advisory board from BoehringerIngelheim and BMS/Pfizer. Dr Bonati has received funding from the Swiss National Science Foundation, the University of Basel, and the Swiss Heart Foundation, received travel honoraria from Bayer and served on scientific advisory boards for Bayer. Dr Leys participated during the last 5 years to advisory boards, symposia or trials sponsored by Sanofi Aventis, BMS, AstraZeneca, Boeringher-Ingelheim, Servier, Ebewe, CoLucid Pharm, Brainsgate, Photothera, Lundbeck, GSK, Bayer, Pfizer et Allergan (honorarium paid to Adrinord or research account of the hospital) and was an associated editor of the Journal of Neurology, Neurosurgery and Psychiatry 2004-2010 (personal financial compensation). Dr Cordonnier is a member of advisory boards (Bayer), trials (AstraZeneca, Pfizer, and Medtronic). 
Since 2003 (beginning of the study), relevant to the topic: symposia (Boeringher-Ingelheim; honorarium paid to Adrinord or to the Lille University Hospital. No personal funding). Dr Michel received research grants from the Swiss National Science Foundation, the Swiss Heart Foundation; speaker fees from Boehringer-Ingelheim, Bayer, Covidien, and Stryker; honoraria from scientific advisory boards from Boehringer-Ingelheim, Bayer, Pfizer, Amgen; consulting fees from Pierre-Fabre and Astra-Zeneca; and travel support from Boehringer-Ingelheim and Bayer. All these supports are received by the institution (CHUV) and are used for stroke education and research. Dr Nolte has received funding for travel or speaker honoraria from Bayer, Boehringer-Ingelheim, Takeda and BMS/Pfizer. Dr Ringleb received modest honoraria for lectures and advisory board from Boehringer-Ingelheim. The University Hospital Heidelberg is sponsor of the European Cooperative Acute Stroke Study (ECASS) IV trial, examining the role of recombinant tissue-type plasminogen activator in an extended time-window, which is financed by Boehringer-Ingelheim. Dr Lyrer has served on scientific advisory boards for Bayer, Daiichi-Sankyo, Schering Pharma, and BoehringerIngelheim has received funding for travel or speaker honoraria from Bayer Schering Pharma, Boehringer-Ingelheim, and Shire plc; he has received research support from AstraZeneca, Boehringer-Ingelheim, Sanofi-Aventis, PhotoThera, the Swiss National Science Foundation, and the Swiss Heart Foundation. Dr Tatlisumak had research contracts with Boehringer-Ingelheim, H. Lundbeck A/S, Pfizer, and Bayer. Advisory board memberships: Boehringer-Ingelheim, H. Lundbeck A/S, Pfizer, and Bayer. Dr Nederkoorn has received consulting fees from Boehringer-Ingelheim. Dr Engelter has received funding for travel or speaker honoraria from Bayer and Boehringer-Ingelheim, he has served on scientific advisory boards for Bayer, BoehringerIngelheim, BMS/Pfizer, and Covidien and on the editorial board of Stroke. He has received research support from the Science Funds (Wissenschaftsfonds) of the University Hospital Basel, the Swiss Heart Foundation, and the Swiss National Science Foundation. The other authors report no conflicts.

\section{References}

1. Emberson J, Lees KR, Lyden P, Blackwell L, Albers G, Bluhmki E, et al; Stroke Thrombolysis Trialists' Collaborative Group. Effect of treatment delay, age, and stroke severity on the effects of intravenous thrombolysis with alteplase for acute ischaemic stroke: a meta-analysis of individual patient data from randomised trials. Lancet. 2014;384:1929-1935. doi: 10.1016/S0140-6736(14)60584-5.

2. Bruno A, Shah N, Lin C, Close B, Hess DC, Davis K, et al. Improving modified Rankin Scale assessment with a simplified questionnaire. Stroke. 2010;41:1048-1050. doi: 10.1161/STROKEAHA.109.571562.

3. Sandercock P, Wardlaw JM, Lindley RI, Dennis M, Cohen G, Murray G et al. The benefits and harms of intravenous thrombolysis with recombinant tissue plasminogen activator within $6 \mathrm{~h}$ of acute ischaemic stroke (the third international stroke trial [IST-3]): a randomised controlled trial. Lancet. 2012;379:2352-2363.

4. Foell RB, Silver B, Merino JG, Wong EH, Demaerschalk BM, Poncha F, et al. Effects of thrombolysis for acute stroke in patients with preexisting disability. CMAJ. 2003;169:193-197.
5. Rudd M, Rodgers H, Curless R, Sudlow M, Huntley S, Madhava B, et al. Remote specialist assessment for intravenous thrombolysis of acute ischaemic stroke by telephone. Emerg Med J. 2012;29:704-708. doi: 10.1136/emermed-2011-200582.

6. Engelter ST, Soinne L, Ringleb P, Sarikaya H, Bordet R, Berrouschot J, et al. IV thrombolysis and statins. Neurology. 2011;77:888-895. doi: 10.1212/WNL.0b013e31822c9135.

7. Gensicke H, Zinkstok SM, Roos YB, Seiffge DJ, Ringleb P, Artto V, et al. IV thrombolysis and renal function. Neurology. 2013;81:1780-1788. doi: 10.1212/01.wnl.0000435550.83200.9e.

8. Zinkstok SM, Engelter ST, Gensicke H, Lyrer PA, Ringleb PA, Artto $\mathrm{V}$, et al. Safety of thrombolysis in stroke mimics: results from a multicenter cohort study. Stroke. 2013;44:1080-1084. doi: 10.1161/ STROKEAHA.111.000126.

9. Scheitz JF, Seiffge DJ, Tütüncü S, Gensicke H, Audebert HJ, Bonati $\mathrm{LH}$, et al. Dose-related effects of statins on symptomatic intracerebral hemorrhage and outcome after thrombolysis for ischemic stroke. Stroke. 2014;45:509-514. doi: 10.1161/STROKEAHA.113.002751.

10. Lyden P, Brott T, Tilley B, Welch KM, Mascha EJ, Levine S, et al. Improved reliability of the NIH Stroke Scale using video training. NINDS TPA Stroke Study Group. Stroke. 1994;25:2220-2226.

11. Fluri F, Hatz F, Voss B, Lyrer PA, Engelter ST. Restenosis after carotid endarterectomy: significance of newly acquired risk factors. Eur $J$ Neurol. 2010;17:493-498. doi: 10.1111/j.1468-1331.2009.02858.x.

12. Karlinski M, Kobayashi A, Czlonkowska A, Mikulik R, Vaclavik D, Brozman M, et al; Safe Implementation of Treatments in Stroke-Eastern Europe (SITS-EAST) Investigators. Role of preexisting disability in patients treated with intravenous thrombolysis for ischemic stroke. Stroke. 2014;45:770-775. doi: 10.1161/STROKEAHA.113.003744.

13. Hacke W, Kaste M, Fieschi C, von Kummer R, Davalos A, Meier D, et al. Randomised double-blind placebo-controlled trial of thrombolytic therapy with intravenous alteplase in acute ischaemic stroke (ECASS II). Second European-Australasian Acute Stroke Study Investigators. Lancet. 1998;352:1245-1251.

14. Kammersgaard LP, Jørgensen HS, Reith J, Nakayama H, Pedersen PM, Olsen TS; Copenhagen Stroke Study. Short- and long-term prognosis for very old stroke patients. The Copenhagen Stroke Study. Age Ageing. 2004;33:149-154. doi: 10.1093/ageing/afh052.

15. Alshekhlee A, Li CC, Chuang SY, Vora N, Edgell RC, Kitchener JM, et al. Does dementia increase risk of thrombolysis?: a case-control study. Neurology. 2011;76:1575-1580. doi: 10.1212/WNL.0b013e3182190d37.

16. Saposnik G, Kapral MK, Cote R, Rochon PA, Wang J, Raptis S, et al. Is pre-existing dementia an independent predictor of outcome after stroke? A propensity score-matched analysis. J Neurol. 2012;259:2366-2375. doi: 10.1007/s00415-012-6508-4.

17. Suarez JI, Sunshine JL, Tarr R, Zaidat O, Selman WR, Kernich C, et al. Predictors of clinical improvement, angiographic recanalization, and intracranial hemorrhage after intra-arterial thrombolysis for acute ischemic stroke. Stroke. 1999;30:2094-2100.

18. Mishra NK, Ahmed N, Davalos A, Iversen HK, Melo T, Soinne L, et al; SITS and VISTA Collaborators. Thrombolysis outcomes in acute ischemic stroke patients with prior stroke and diabetes mellitus. Neurology. 2011;77:1866-1872. doi: 10.1212/WNL.0b013e318238ee42.

19. Fearon P, McArthur KS, Garrity K, Graham LJ, McGroarty G, Vincent S, et al. Prestroke modified rankin stroke scale has moderate interobserver reliability and validity in an acute stroke setting. Stroke. 2012;43:31843188. doi: 10.1161/STROKEAHA.112.670422. 